\title{
Evaluation Study on Viable IFS Models in Visakhapatnam District of Andhra Pradesh
}

\author{
D. Uma Maheswara Rao*, N. Sathibabu, K. Tejeswara Rao, \\ N. Rajkumar, V. Gouri and G. Manasa \\ Krishi Vigan Kendra (ANGRAU), Kondempudi, Visakahpatnam dt, AP, India \\ *Corresponding author
}

\section{Ke ywords \\ IFS Models, \\ Visakhapatnam, \\ Sugarcane + \\ Groundnut+Tomato}

Article Info

Accepted:

28 November 2020

Available Online:

10 December 2020

\section{A B S T R A C T}

Visakhapatnam district has two distinct geographical sub divisions covering 32 mandals under Plain division and 11 mandals under agency division. The district 4.56 lakh farmers, They are predominantly small, Marginal farmers covering $95 \%$ of the total farming community of the district. With limited farm holdings these marginal and small farmers are bund to adopt Integrated Farming Systems approach to get sustained income to support their families. Visakhapatnam dt has 8 different farming situations covering 3 lakh hectares of total area. In the present study it was observed several integrated farming systems adopted by the farmers based on the resources available under different farming situations. Nearly 60 different systems were analysed, Prioritized and ranked based on their B:C ratio under 6 major farming situations. Top 3 farming systems from each of 6 farming situations were prioritized and Picked up and coming to a total 18 farming systems. These farming systems predominantly having major field crops, Vegetable crops, Dairy and Poultry as combination of components. These 18 farming systems were further analyzed and found that 1 . Sugarcane + Groundnut+Tomato 2 . Paddy + Groundnut falling under Tank fed Red sand soils farming systems model ranks $1^{\text {st }} \& 3^{\text {rd }}$ respectively and Tomato+Ridge gourd+Dairy falling under Bore well based Red Sandy loams farming systems model ranks $2^{\text {nd }}$.as per B:C ratio. From the above study, it was observed that Under Rain fed situations more diversified crops are adopted compared to canal fed system. Paddy or Sugarcane based system mostly has includes Dairy as one of the components and which is mostly found profitable and enhances overall profitability of the system. Under Rain fed system due to lack of assured irrigation, the farmers are adopting combination of vegetable crops like leafy vegetables, vegetables which are completed in a short duration. The evolved successful IFS models across the major farming systems are to be preserved to provide sustainable in situ livelihoods to the farming communities spreading in remote corners of the district.

\section{Introduction}

"Integrated Farming" (IF) is a whole farm management system which aims to bring more sustainability in agriculture. It is a dynamic approach which can be applied to any farming system around the world. It involves attention to impart continuous improvement in all areas of a farming business through informed management 
processes. Integrated Farming combines the best of modern tools and technologies with traditional practices according to a given site and situation.

The International Organization of Biological Control (IOBC) describes Integrated Farming as a farming system where high quality food, feed, fiber and renewable energy are produced by using resources such as soil, water, air and nature as well as regulating factors contribute to the farm sustainably and with as little polluting inputs as possible.

Particular emphasis is placed on a holistic management approach looking at the whole farm as inter linked unit, on the fundamental role and function of agro-ecosystems, on nutrient cycles which are balanced and adapted to the demand of the crops, health and welfare of all livestock on the farm. Preserving and enhancing soil fertility, maintaining and improving a diverse environment and the adherence to ethical and social criteria are indispensable basic elements. Crop protection takes into account all biological, technical and chemical methods, which then are balanced carefully and with the objective to protect the environment, to maintain profitability of the business and fulfil social requirements.

In recent years, food security, livelihood security, water security as well as natural resources conservation and environment protection have emerged as major issues worldwide. Developing countries are struggling to deal with these issues and also have to contend with the dual burden of climate change and globalization.

It has been accepted by decision makers across the globe that sustainable development is the only way to promote rational utilization of resources and environmental conservation without hampering economic growth. Different countries around the world are promoting sustainable development through sustainable agricultural practices, which will help them in addressing socio-economic as well as environmental issues simultaneously.

Within the broad concept of sustainable agriculture "Integrated Farming Systems" hold special position as in this system nothing is wasted, the by-product of one system becomes the input for other. Integrated farming is an integrated approach to farming as compared to existing monoculture approaches. It refers to agricultural systems that integrate livestock and crop production. Moreover, the system help poor small farmers, who have very small land holding for crop production and a few heads of livestock to diversify farm production, increase cash income, improve quality and quantity of food produced and exploitation of unutilized resources.

Components of integration in a farming system are trees on bunds, wind breaks, silvipasture system, agro-horticulture system, block plantations, economic shrubs, live fences, crops with green leaf manure species (mixed/intercrops), integrated animal based systems (fisheries, dairy, piggery, small ruminants, poultry, apiary).

\section{Visakhapatnam district profile}

Visakhapatnam District is one of the North Eastern Coastal districts of Andhra Pradesh and it lies between $17^{\circ}-15^{\prime}$ and $18^{\circ}-32^{\prime}$ Northern latitude and $18^{\circ}-54^{\prime}$ and $83^{\circ}-30^{\prime}$ in Eastern longitude. It is bounded on the North partly by the Orissa State and partly by Vizianagaram District, on the South by East Godavari District, on the West by Orissa State and on the East by Bay of Bengal.

\section{Physical features}

The District presents two distinct Geographic divisions. The strip of the land along the coast 
and the interior called the plains division and hilly area of the Eastern Ghats flanking it on the North and West called the Agency Division. The Agency Division consists of the hilly regions covered by the Eastern Ghats with an altitutde of about 900 metres dotted by several peaks exceeding 1200 metres.

\section{Rainfall}

The District receives an annual normal rainfall of $1202 \mathrm{~mm}$.

Visakhapatnam district has 8 major farming situations spreading in an area of around 3 lakh hectares. Majority of the farmers in the district are small and marginal farmers. Majoritv farmers are adopting integrated farming systems model. They are deriving viable and sustainable livelihoods through these IFS models.

Demographic and census details of Visakhapatnam dt

Population data (Give classified population data of the district (as per 2011 census)

i)Rural : 22.51 lakhs $(52.49 \%$ of total population)

ii)Urban : 20.37 Lakhs $(47.51 \%$ of total population)

Farming population: 4.56 Lakhs (2011 Census)

Agrl. Labourers: 8.55 Lakhs (2011 Census)

Total population of the district:Total : 42.88 Lakhs (2011 Census)

Male : 21.41 Lakhs

Female : 21.47 Lakhs

Marginal farmers : 3.39 Lakhs

Small farmers : 0.69 Lakhs
Semi Medium farmers : 0.35 Lakhs

Medium farmers : 0.12 Lakhs

Large farmers : 0.012 Lakhs

Out of the above farmers in the District, the Hilly farmers are: 0.87 Lakhs

The main objectives of this study include to study the sustainable Integrated Farming System models evolved by the farmers of the district. And to identify the most economically viable/Profitable cropping situations adopted by the farmers of the district.

\section{Locale of the study}

The study was conducted in rural Visakhapatnam Dt. The data was collected from 120 farmers spreading 6 villages of 3 mandals across the district like Santha palem, R.Y. Agraharam, Gondupalem (K.Kotapadu madal), Gouripatnam, R. (Chodavaram mandal) and Kothapenta (Devarapalle mandal).

\section{Materials and Methods}

To assess the viable IFS models evolved by the farmers of Visakhapatnam district. The data collected from 120 farmers by individual questionnaire method. The data got analyzed using statistical methods like Benefit cost ratio $\&$ ranking methods. The analyzed data was interpreted by using Bar charts and graphs.

Type of farming (Farming systems): The major farming systems prevailed in Visakhapatanm District are given in Table 1.

Farming situation wise profitable IFS models identified in the district as follows

\section{Well based red sandy loams}

There are 11 different farming systems identified under Well based red sandy loams, 
They were listed, analyzed as per their benefit cost ratio and ranked as mentioned in Table 2. Out of the 11 Integrated Farming Systems identified, Tomato+Ridge gourd+Dairy is ranked Ist with $\mathrm{B}: \mathrm{C}$ ratio 3.48 followed Paddy+Tomato+Brinjal+Dairy ranked $2^{\text {nd }}$ with B:C ratio 2.69 and Paddy+Maize+ Tomato+Bhendi ranked $3^{\text {rd }}$ with $\mathrm{B}: \mathrm{C}$ ratio 2.60 under well based Red Sandy loams farming situation. The Production constraints identified under the above situation are listed as below with probable suggestions given by farmers to over come the constraints.

\section{Production constraints in IFS models as perceived by the farmers}

\section{Well based Red Sandy loams}

Since irrigation is a problem, and the major crops are mainly rainfed, paddy cultivation is difficult.

Lack of awareness on different varieties.

Integrated management is not followed due to lack of knowledge.

Not following the suggestions followed by agricultural officials.

Practices of unwanted activities, Eg: Chloro dusting.

Not following proper spacing in crops.

Lack of knowledge on subsidies and schemes.

Suggestions for strengthening of IFS models as perceived by the farmers (Fig. 1)

\section{Well based red sandy loams:}

To follow scrupulously suggestions of the agricultural officials.

Actively participation in awareness programmes.

Take memberships in FPO's.

Following the management practices at right time.

Proper utilization of Government schemes.

Agricultural produce should be marketed through regulated market channel.
Rainfed Red Sandy loams (96,656 ha) wise profitable IFS models in the district

There are 13 different farming systems identified under Rainfed Red Sandy loams, They were listed, analyzed as per their benefit cost ratio and ranked as mentioned in Table 3.

Out of the 13 Integrated Farming Systems identified, Paddy+Groundnut+Chilli+Bhendi is ranked Ist with $\mathrm{B}: \mathrm{C}$ ratio 3.04 followed Paddy+Dairy ranked $2^{\text {nd }}$ with $B: C$ ratio 2.53 and Paddy+Groundnut+Sesame+Brinjal+ Chilli ranked $3^{\text {rd }}$ with $\mathrm{B}: \mathrm{C}$ ratio 2.18 under well based Red Sandy loams farming situation. The Production constraints identified under the above situation are listed as below with probable suggestions given by farmers to overcome the constraints (Fig. 2).

\section{Rainfed Red sandy soils}

Constraints:

1.Lack of awareness on new varieties, 2. Lack of knowledge on IPM practices.

3.Lack of knowledge on Rodent management 4.Lack of knowledge on micro nutrient management

5.Lack of knowledge on subsidies and schemes.

6.Wild bore attack to Horticultural crops.

7.Lack of transport facility to marketing of agri products.

8. Lack of awareness on weed management in different crops and spending lot money on labour.

9.Lack of awareness on different agronomic practices in different crops.

\section{Rainfed red sandy soils}

\section{Suggestions}

Providing resistant varieties of seeds to farmers on subsidy.

Active participation in awareness 
programmes.

Following the management practices at right time.

Proper utilization of government schemes.

Taking proper suggestions from agricultural officers.

Aqire knowledge on IPM practices.

Provide better marketing facilities.

Introduce better control measures for rodent and wild boar management.

Develop permenant village information corner to address seasonal agricultural problems.

Canal Based clay soils Farming situation wise profitable IFS models in the district

There are 9 different farming systems identified under Canal Based clay soils Farming situation. They were listed, analyzed as per their benefit cost ratio and ranked as mentioned Table 4.

Out of the 9 Integrated Farming Systems identified, Paddy-Pulses-Sunhemp is ranked Ist with $\mathrm{B}: \mathrm{C}$ ratio 1.66 followed by PaddyPulses-Bhendi ranked $2^{\text {nd }}$ with $\mathrm{B}: \mathrm{C}$ ratio 1.6 and Paddy-Sugracane-Dairy ranked $3^{\text {rd }}$ with $\mathrm{B}: \mathrm{C}$ ratio 1.5 under Canal Based clay soils Farming situation farming situation. The Production constraints identified under the above situation are listed as below with probable suggestions given by farmers to overcome the constraints (Fig. 3).

\section{Constraints}

\section{Canal based clay soils}

\section{Lack of awareness on ICM}

Not following the advises of Dept of agriculture..

Lack of belief on modern technology..

Seed supply is not done timely..

Labour shortage.

Marketing facilitate is not properly established.
Marketing prices are not remunerative.

Canal Based clay soils, (Kotha penta, Devarapalle mandal)

\section{Suggestions}

Create awareness.

Create confidence.

Seed should be supplied in time..

Increase marketing facility

Provide profitable price for the agricultural produce.

\section{Rainfed Red loamy soils Farming situation wise profitable IFS models in the district}

There are 11 different farming systems identified under Rainfed Red loamy soils Farming situation. They were listed, analyzed as per their benefit cost ratio and ranked as mentioned Table 5.

Out of the 11 Integrated Farming Systems identified, Paddy-Papaya-Vegetables-Dairy is ranked Ist with B:C ratio 2.66 followed by Paddy-Sugarcane -Dairy ranked $2^{\text {nd }}$ with B:C ratio 2.56 and Paddy-Greengram-SeasmeVegetables ranked $3^{\text {rd }}$ with $\mathrm{B}: \mathrm{C}$ ratio 2.51 under Rainfed Red loamy soils Farming situation farming situation. The Production constraints identified under the above situation are listed as below with probable suggestions given by farmers to overcome the constraints.

\section{Rainfed red loamy soils}

\section{Constraints}

1.Illiteracy among the farmers.

2.Lack of awareness on various government schemes.

3.Lack of enough technical experts to educate the farmers.

4.To poor managerial ability to take up profitable IFS models.

5.Lack of financial ability among farmers to adapt improved technology. 
6.Lack of knowledge on modern pest control measures.

7.lack of proper waste management among the farmers.

VI. Rainfed Red loamy soils, R. NARSAPURAM

\section{Suggestions}

1.Organisation of proper training and awareness proper to the farmers.

2.Proper timely financial assistance to the farmers.

3.Provide assistance to farmers to adapt to profitable IFS models.

4.Expose the farmers to profitable IFS models.

Tank fed Red sandy soils (36,538 ha) Farming situation wise profitable IFS models in the district

There are 10 different farming systems identified under Tank fed Red sandy soils Farming situation. They were listed, analyzed as per their benefit cost ratio and ranked as mentioned in Table 6 .

Out of the 10 Integrated Farming Systems identified, Sugarcane-Groundnut-Tomato is ranked Ist with B:C ratio 6 followed by Paddy- Groundnut ranked $2^{\text {nd }}$ with B:C ratio 3.2 and Sugarcane-Groundnut-Chilli ranked $3^{\text {rd }}$ with B:C ratio 3.1 under Tank fed Red sandy soils Farming situation farming situation.

The Production constraints identified under the above situation are listed as below with probable suggestions given by farmers to overcome the constraints (Fig. 4).

\section{Tank fed red sandy soils}

\section{Constraints}

1.lack of awareness on new varieties. 2.lack of knowledge on IPM practices.
3.Timely vaccinations are not provided to cattle.

4.Lack of knowledge on drip and sprinkler irrigation.

5.Lack of knowledge on subsidies and schemes.

\section{Tank fed red sandy soils}

\section{Suggestions}

1.Provide resistant varieties of seeds to farmers.

2.Active participation in awareness programmes

3.Create awareness on profitable IFS models.

4.Follow best management practices at right time.

5.Proper utilization of government schemes.

6.Take proper suggestions from agricultural officers.

7.Develop knowlwdge on microirrigation sstems.

Canal based alluvial soils $(13,353$ ha) Farming situation wise profitable IFS models in the district

There are 4 different farming systems identified under Canal based alluvial soils Farming situation. They were listed, analyzed as per their benefit cost ratio and ranked as mentioned in Table 7.

Out of the 4 Integrated Farming Systems identified, Paddy+Pulses+Dairy is ranked Ist with B:C ratio 2.88 followed by Sugarcane+ dairy ranked $2^{\text {nd }}$ with $\mathrm{B}: \mathrm{C}$ ratio 2.83 and Paddy+Paddy+Dairy ranked $3^{\text {rd }}$ with $B: C$ ratio 2.75 under Canal based alluvial soils Farming situation farming situation. The Production constraints identified under the above situation are listed as below with probable suggestions given by farmers to overcome the constraints. 
Canal fed - alluvial soils

\section{Constraints}

Lack of awareness profitable cropping pattern.

Soil testing is not followed.

Lack of awareness on high yielding varieties.
Lack of awareness on seed treatment.

Usage of high seed rate

Use of high dose of fertilizers and pesticides.

Lack of awareness about timely plant protection measures

Do not follow the instructions of Dept of Agriculture.

Market prices are not remunerative..

Table.1 Type of farming (Farming systems): The major farming systems prevailed in

Visakhapatanm. District are as follows

\begin{tabular}{|l|l|r|}
\hline \multicolumn{1}{|c|}{ Farming situation } & \multicolumn{1}{|c|}{ Description } & Area in ha \\
\hline Farming Situation - 1 & $\begin{array}{l}\text { Canal Irrigation - Alluvial } \\
\text { Soils }\end{array}$ & $\mathbf{1 3 , 3 5 3}$ \\
\hline Farming Situation - 2 & Canal Irrigation - Clay Loams & $\mathbf{1 8 , 5 9 0}$ \\
\hline Farming Situation - 3 & Irrigated Check Dams & $\mathbf{2 1 , 9 8 9}$ \\
\hline Farming Situation - 4 & $\begin{array}{l}\text { Well Irrigation - Red Clay } \\
\text { Loams }\end{array}$ & $\mathbf{1 0 , 2 8 9}$ \\
\hline Farming Situation - 5 & Tank Irrigation - Red Loams & $\mathbf{3 6 , 5 3 9}$ \\
\hline Farming Situation - 6 & Rain fed - Red Clay Loams & $\mathbf{9 7 , 6 3 0}$ \\
\hline Farming Situation - 7 & Rain fed - Red Sandy Loams & $\mathbf{9 6 , 6 5 6}$ \\
\hline Farming Situation - 8 & Rain fed - Coastal Sandy Soils & $\mathbf{6 , 8 5 6}$ \\
\hline & Total & $\mathbf{3 , 0 1 , 9 0 2}$ \\
\hline
\end{tabular}

Table.2 Well based red sandy loams

\begin{tabular}{|c|c|c|c|c|c|c|c|}
\hline S.No & $\begin{array}{l}\text { Farming } \\
\text { situation }\end{array}$ & Farming systems identified & $\begin{array}{l}\text { Cost of } \\
\text { cultivation }\end{array}$ & $\begin{array}{l}\text { Gross } \\
\text { returns }\end{array}$ & $\begin{array}{c}\text { Net } \\
\text { returns }\end{array}$ & $\begin{array}{l}\text { B.C } \\
\text { Ratio }\end{array}$ & $\begin{array}{l}\text { Ran } \\
\mathbf{k}\end{array}$ \\
\hline 1 & \multirow{11}{*}{$\begin{array}{c}\text { well based } \\
\text { Red Sandy } \\
\text { loams } \\
(10,289)\end{array}$} & Paddy+Tomato+Brinjal+Dairy & 45800 & 123440 & 77640 & 2.69 & 2 \\
\hline 2 & & Paddy+Brinjal+Tomato & 32200 & 77400 & 45400 & 2.40 & 6 \\
\hline 3 & & Tomato+Brinjal & 11000 & 20900 & 9900 & 1.90 & 11 \\
\hline 4 & & Tomato+Paddy & 38788 & 96970 & 58182 & 2.5 & 4 \\
\hline 5 & & Tomato+Paddy+Brinjal+Dairy & 105550 & 209010 & 103460 & 1.98 & 10 \\
\hline 6 & & Tomato+Ridge gourd+Dairy & 40000 & 139550 & 99550 & 3.48 & 1 \\
\hline 7 & & Cauliflower+Bhendi+Chilli+Dairy & 62000 & 128850 & 66850 & 2.07 & 9 \\
\hline 8 & & Paddy+Tomato+Brinjal+Bhendi & 6000 & 129480 & 69480 & 2.158 & 7 \\
\hline 9 & & Paddy+Capicum+Tomato+Dairy & 117760 & 289680 & 171924 & 2.46 & 5 \\
\hline 10 & & Chilli+Cauliflower+Dairy & 69160 & 148000 & 78840 & 2.14 & 8 \\
\hline 11 & & Paddy+Maize+Tomato+Bhendi & 57770 & 150200 & 92430 & 2.60 & 3 \\
\hline
\end{tabular}


Table.3 Rainfed Red Sandy loams (96,656 ha) wise profitable IFS models in the district

\begin{tabular}{|c|c|c|c|c|c|c|c|}
\hline S.No. & $\begin{array}{l}\text { Farming } \\
\text { situation }\end{array}$ & Farming systems identified & $\begin{array}{l}\text { Cost of } \\
\text { cultivation }\end{array}$ & $\begin{array}{l}\text { Gross } \\
\text { return } \\
\text { s }\end{array}$ & $\begin{array}{l}\text { Net } \\
\text { returns }\end{array}$ & $\begin{array}{l}\text { B:C } \\
\text { Ratio }\end{array}$ & Rankingnk \\
\hline 1 & \multirow{7}{*}{$\begin{array}{l}\text { Rainfed } \\
\text { Red } \\
\text { Sandy } \\
\text { loams } \\
(96,656 \\
\text { ha) }\end{array}$} & Paddy+Groundnut+Chilli+Bhendi & 25000 & 76000 & 51000 & 3.04 & 1 \\
\hline 3 & & Paddy+Groundnut+Sesame+Brinjal+Chilli & 65000 & 142000 & 77000 & 2.184 & 3 \\
\hline 4 & & Paddy+ Bhendi+ Dairy & 60000 & 128000 & 68000 & 2.13 & 4 \\
\hline 7 & & Paddy+Groundnuti+Chilli+Dairy & 58000 & 117200 & 59200 & 2.020 & 7 \\
\hline 8 & & Paddy+Sugarcane+Bottle gourd & 63000 & 121500 & 58500 & 1.93 & 8 \\
\hline 9 & & $\begin{array}{l}\text { Paddy+Sugarcane Groundnut+Tomato+Bottle } \\
\text { gourd }\end{array}$ & 68000 & 130500 & 62500 & 1.91 & 9 \\
\hline 10 & & $\begin{array}{l}\text { Paddy+Sugarcane }+ \text { Bhendi+Bottle } \\
\text { gourd+Dairy }\end{array}$ & 94000 & 177000 & 83000 & 1.88 & 10 \\
\hline
\end{tabular}


Table.4 Canal based clay soils Farming situation wise profitable IFS models in the district

\begin{tabular}{|c|c|c|c|c|c|c|c|}
\hline S,No & $\begin{array}{l}\text { Farming } \\
\text { situation }\end{array}$ & $\begin{array}{l}\text { Farming systems } \\
\text { identified }\end{array}$ & $\begin{array}{l}\text { Cost of } \\
\text { cultivation }\end{array}$ & Gross returns & Net returns & B.C Ratio & Rank \\
\hline \multirow[t]{2}{*}{1} & \multirow{10}{*}{$\begin{array}{l}\text { Canal } \\
\text { Based } \\
\text { clay soils } \\
\text { Farming } \\
\text { situation }\end{array}$} & $\begin{array}{l}\text { Paddy-Pulses- } \\
\text { Groundnut }\end{array}$ & 20000 & 48000 & 28000 & 1.4 & 5 \\
\hline & & $\begin{array}{l}\text { Paddy-Sugracane- } \\
\text { Dairy }\end{array}$ & 50000 & 120000 & 75000 & 1.5 & 3 \\
\hline 2 & & Paddy-Paddy-Dairy & 55000 & 110000 & 55000 & 1.0 & 10 \\
\hline 3 & & $\begin{array}{l}\text { Sugarcane- } \\
\text { Sugarcane-Dairy }\end{array}$ & 50000 & 120000 & 70000 & 1.42 & 4 \\
\hline 4 & & $\begin{array}{l}\text { Paddy-Pulses- } \\
\text { Groundnut-Dairy }\end{array}$ & 40000 & 85000 & 45000 & 1.125 & 9 \\
\hline 5 & & $\begin{array}{l}\text { Paddy-Paddy- } \\
\text { Sesame }\end{array}$ & 35000 & 75000 & 40000 & 1.14 & 8 \\
\hline 6 & & $\begin{array}{l}\text { Paddy-Paddy- } \\
\text { Sesame }\end{array}$ & 19000 & 45000 & 26000 & 1.36 & 6 \\
\hline 7 & & $\begin{array}{l}\text { Paddy-Pulses- } \\
\text { Sunhemp }\end{array}$ & 15000 & 40000 & 25000 & 1.66 & 1 \\
\hline 8 & & $\begin{array}{l}\text { Sugarcane-Pulses- } \\
\text { Dairy }\end{array}$ & 45000 & 100000 & 55000 & 1.22 & 7 \\
\hline 9 & & $\begin{array}{l}\text { Paddy-Pulses- } \\
\text { Bhendi }\end{array}$ & 25000 & 65000 & 40000 & 1.60 & 2 \\
\hline
\end{tabular}

Table.5 Rainfed red loamy soils farming situation wise profitable IFS models in the district

\begin{tabular}{|c|c|c|c|c|c|c|c|}
\hline S,No & $\begin{array}{l}\text { Farming } \\
\text { situation }\end{array}$ & Farming systems identified & $\begin{array}{l}\text { Cost of } \\
\text { cultivation }\end{array}$ & $\begin{array}{l}\text { Gross } \\
\text { returns }\end{array}$ & $\begin{array}{c}\text { Net } \\
\text { returns }\end{array}$ & $\begin{array}{c}\text { B.C } \\
\text { Ratio }\end{array}$ & Ran \\
\hline 1 & \multirow{10}{*}{$\begin{array}{l}\text { Rainfed } \\
\text { Red } \\
\text { loamy } \\
\text { soils } \\
(97,630 \\
\text { ha) }\end{array}$} & Paddy- Tomato-Dairy & 60,570 & $\begin{array}{c}1,16,38 \\
0\end{array}$ & 55,760 & 1.92 & 7 \\
\hline 2 & & Sugarcane- Vegetable-Dairy & 53,620 & $\begin{array}{c}1,30,52 \\
0\end{array}$ & 76,900 & 2.43 & 4 \\
\hline 3 & & $\begin{array}{l}\text { Paddy-Green-Gram- } \\
\text { Sesame-Vegetable }\end{array}$ & 40,120 & $\begin{array}{c}1,00,75 \\
0\end{array}$ & 60,630 & 2.51 & 3 \\
\hline 4 & & Sugarcane- Maize-Dairy & 58,620 & $1,37,530$ & 78,910 & 2.34 & 5 \\
\hline 5 & & Paddy-Sugarcane-Dairy & 62,650 & $1,60,560$ & 97,910 & 2.56 & 2 \\
\hline 6 & & Vegetable-Dairy & 40,060 & 68,080 & 28,020 & 1.69 & 9 \\
\hline 7 & & Maize-Vegetable-Dairy & 53,070 & 89,050 & 35,980 & 1.67 & 10 \\
\hline 8 & & $\begin{array}{l}\text { Maize-Greengram- } \\
\text { Vegetable- Dairy }\end{array}$ & 68,120 & $1,03,740$ & 35,620 & 1.52 & 11 \\
\hline 9 & & Paddy-Maize-Tomato-Dairy & 53,580 & $1,37,070$ & 63,490 & 1.86 & 8 \\
\hline 10 & & Paddy-Groundnut-Dairy & 65,120 & $1,34,120$ & 69,000 & 2.05 & 6 \\
\hline 11 & & $\begin{array}{l}\text { Paddy-Papaya-Vegetables- } \\
\text { Dairy }\end{array}$ & 72,090 & $1,92,120$ & $1,20,030$ & 2.66 & 1 \\
\hline
\end{tabular}


Table.6 Tank fed Red sandy soils (36,538 ha) Farming situation wise profitable IFS models in the district

\begin{tabular}{|c|c|c|c|c|c|c|c|}
\hline S,No & $\begin{array}{l}\text { Farming } \\
\text { situation }\end{array}$ & $\begin{array}{l}\text { Farming systems } \\
\text { identified }\end{array}$ & $\begin{array}{l}\text { Cost of } \\
\text { cultiva } \\
\text { tion }\end{array}$ & $\begin{array}{l}\text { Gross } \\
\text { returns }\end{array}$ & $\begin{array}{l}\text { Net } \\
\text { returns }\end{array}$ & $\begin{array}{l}\text { B.C } \\
\text { Ratio }\end{array}$ & Rank \\
\hline \multirow[t]{2}{*}{1} & \multirow{11}{*}{$\begin{array}{l}\text { Tank fed Red } \\
\text { sandy soils } \\
(36,538 \text { ha) }\end{array}$} & Paddy- Groundnut & 56000 & 180000 & 124000 & 3.2 & 2 \\
\hline & & $\begin{array}{l}\text { Sugarcane- } \\
\text { Groundnut- } \\
\text { Tomato }\end{array}$ & 36000 & 218000 & 182000 & 6 & 1 \\
\hline \multirow[t]{2}{*}{2} & & $\begin{array}{l}\text { Sugarcane- } \\
\text { Groundnut- } \\
\text { Sesame }\end{array}$ & 25000 & 70000 & 50000 & 2.8 & 4 \\
\hline & & $\begin{array}{l}\text { Sugarcane- } \\
\text { Groundnut-Chilli }\end{array}$ & 45000 & 95000 & 50000 & 3.1 & 3 \\
\hline 3 & & $\begin{array}{l}\text { Paddy-Tomato- } \\
\text { Brinjal-Dairy }\end{array}$ & 105550 & 209010 & 103460 & 1.98 & 9 \\
\hline 5 & & Paddy-Dairy & 58000 & 128000 & 70000 & 1.20 & 10 \\
\hline 6 & & $\begin{array}{l}\text { Sugarcane- } \\
\text { Cucumber }\end{array}$ & 29000 & 13500 & 20500 & 2.4 & 6 \\
\hline 7 & & Paddy- & 30000 & 58000 & 28000 & 2.01 & 8 \\
\hline 8 & & $\begin{array}{l}\text { Blackgram- } \\
\text { Groundnut }\end{array}$ & & & & & \\
\hline 9 & & $\begin{array}{l}\text { Paddy-Tomato- } \\
\text { Dairy }\end{array}$ & 117760 & 289680 & 171924 & 2.46 & 5 \\
\hline 10 & & Paddy-Sugarcane & 24000 & 55600 & 31600 & 2.31 & 7 \\
\hline
\end{tabular}

Table.7 Canal based alluvial soils (13,353 ha) Farming situation wise profitable IFS models in the district

\begin{tabular}{|l|l|l|l|l|l|l|l|}
\hline $\begin{array}{l}\text { S. } \\
\text { No }\end{array}$ & $\begin{array}{l}\text { Farming } \\
\text { situation }\end{array}$ & $\begin{array}{c}\text { Farming systems } \\
\text { identified }\end{array}$ & $\begin{array}{l}\text { Cost of } \\
\text { cultivation }\end{array}$ & Gross returns & $\begin{array}{c}\text { Net } \\
\text { returns }\end{array}$ & $\begin{array}{l}\text { B.C } \\
\text { Ratio }\end{array}$ & Ran \\
\hline $\mathbf{1}$ & $\begin{array}{l}\text { Canal } \\
\text { based } \\
\text { alluvial }\end{array}$ & Sugarcane+ dairy & 42400 & 120300 & 77900 & 2.83 & 2 \\
\hline $\mathbf{2}$ & Paddy+Seasme+Dairy & 33500 & 98000 & 270200 & 172000 & 2.75 & 3 \\
\hline $\mathbf{3}$ & $\begin{array}{l}\text { soils } \\
\text { (13,353 } \\
\text { ha) }\end{array}$ & $\begin{array}{l}\text { Paddy+ } \\
\text { Paddy+Paddy+Dairy } \\
\text { Pulses+Dairy }\end{array}$ & 75000 & 216000 & 141000 & 2.88 & 1 \\
\hline $\mathbf{4}$ & & & & & \\
\hline
\end{tabular}


Table.8 Dominate IFS models under six major farming situations in the district

\begin{tabular}{|c|c|c|}
\hline S,No & Major Farming situation & Dominant Integrated Farming System models \\
\hline \multirow[t]{3}{*}{1} & \multirow[t]{3}{*}{ Rainfed Red Sandy loams } & 1. Paddy+Groundnut+Chilli+Bhendi \\
\hline & & 2. Paddy+Dairy \\
\hline & & 3.Paddy+Groundnut+Sesame+Brinjal+Chilli \\
\hline \multirow[t]{3}{*}{2} & \multirow[t]{3}{*}{ Canal Based alluvial soils } & 1. Paddy+Pulses+Dairy \\
\hline & & 2. Sugarcane+ dairy \\
\hline & & 3. Paddy+Paddy+Dairy \\
\hline \multirow[t]{3}{*}{3} & \multirow[t]{3}{*}{ Rainfed Red loamy soils } & 1. Paddy-Papaya-Vegetables- Dairy \\
\hline & & 2. Paddy-Sugarcane-Dairy \\
\hline & & 3. Paddy-Green-Gram-Sesame-Vegetable \\
\hline \multirow[t]{3}{*}{4} & \multirow[t]{3}{*}{ Tank fed Red sandy soils } & 1. Sugarcane-Groundnut-Tomato \\
\hline & & 2. Paddy- Groundnut \\
\hline & & 3. Sugarcane-Groundnut-Chilli \\
\hline \multirow[t]{3}{*}{5.} & \multirow{3}{*}{$\begin{array}{l}\text { Well based Red Sandy } \\
\text { loams }\end{array}$} & 1. Tomato+Ridge gourd+Dairy \\
\hline & & 2. Paddy+Maize+Tomato+Dairy \\
\hline & & 3. Paddy+Tomato+Brinjal+Dairy \\
\hline \multirow[t]{3}{*}{6} & Canal based alluvial soils & 1.Paddy+Pulses+Dairy \\
\hline & & 2. Sugarcane+ dairy \\
\hline & & 3. Paddy+Paddy+Dairy \\
\hline
\end{tabular}

Table.9 Successful top 3 IFS models under 6 major farming situations in the district

\begin{tabular}{|c|c|c|c|c|}
\hline S,No & Major Farming situation & $\begin{array}{l}\text { Dominant Integrated Farming } \\
\text { System models }\end{array}$ & B:C Ratio & Rank \\
\hline 1 & $\begin{array}{l}\text { Bore well based Red Sandy } \\
\text { loams }\end{array}$ & 1.Tomato+Ridge gourd+Dairy & 3.48 & II \\
\hline \multirow[t]{2}{*}{2} & Tank fed Red sandy soils & $\begin{array}{l}\text { 2. Sugarcane-Groundnut- } \\
\text { Tomato }\end{array}$ & 6.0 & I \\
\hline & & 3. Paddy- Groundnut & 3.2 & III \\
\hline
\end{tabular}

Table.10 Impressions/conclusion

\begin{tabular}{|c|c|}
\hline S,No & Impressions / Conclusion \\
\hline 1 & $\begin{array}{l}\text { Under Rain fed situations more diversified crops are adopted compared to canal fed } \\
\text { system. }\end{array}$ \\
\hline 2 & $\begin{array}{l}\text { Under canal fed system farmers are tempted to adopt Paddy \& Sugarcane based } \\
\text { cropping systems, which are mostly long standing and non remunerative }\end{array}$ \\
\hline 3 & $\begin{array}{l}\text { Paddy or Sugarcane based system mostly has includes Dairy as one of the component } \\
\text { and which is mostly found profitable and enhances overall profitability of the system. }\end{array}$ \\
\hline 4 & $\begin{array}{l}\text { Under Rain fed system due to lack of assured irrigation, the farmers are adopting } \\
\text { combination of vegetable crops like leafy vegetables, vegetables which are completed } \\
\text { in a short duration. }\end{array}$ \\
\hline
\end{tabular}


Fig.1 Suggestions for strengthening of IFS models as perceived by the farmers

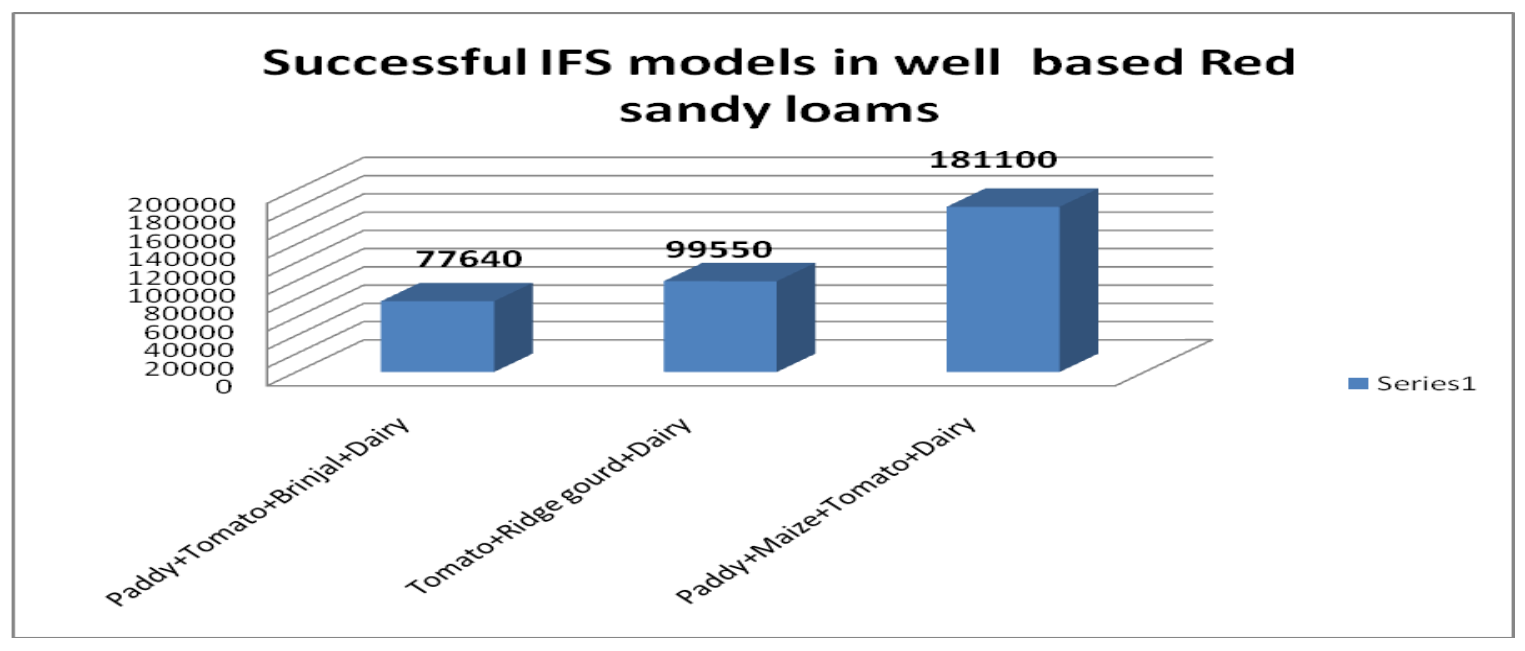

Fig.2

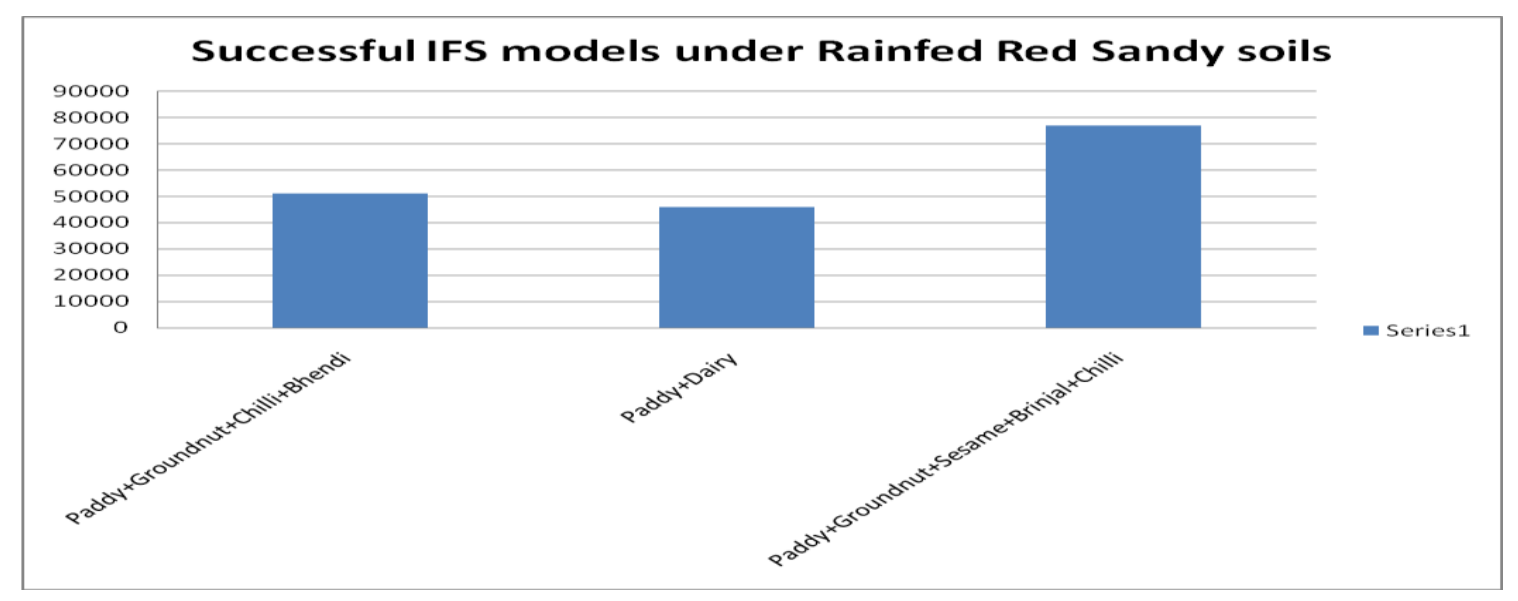

Fig.3

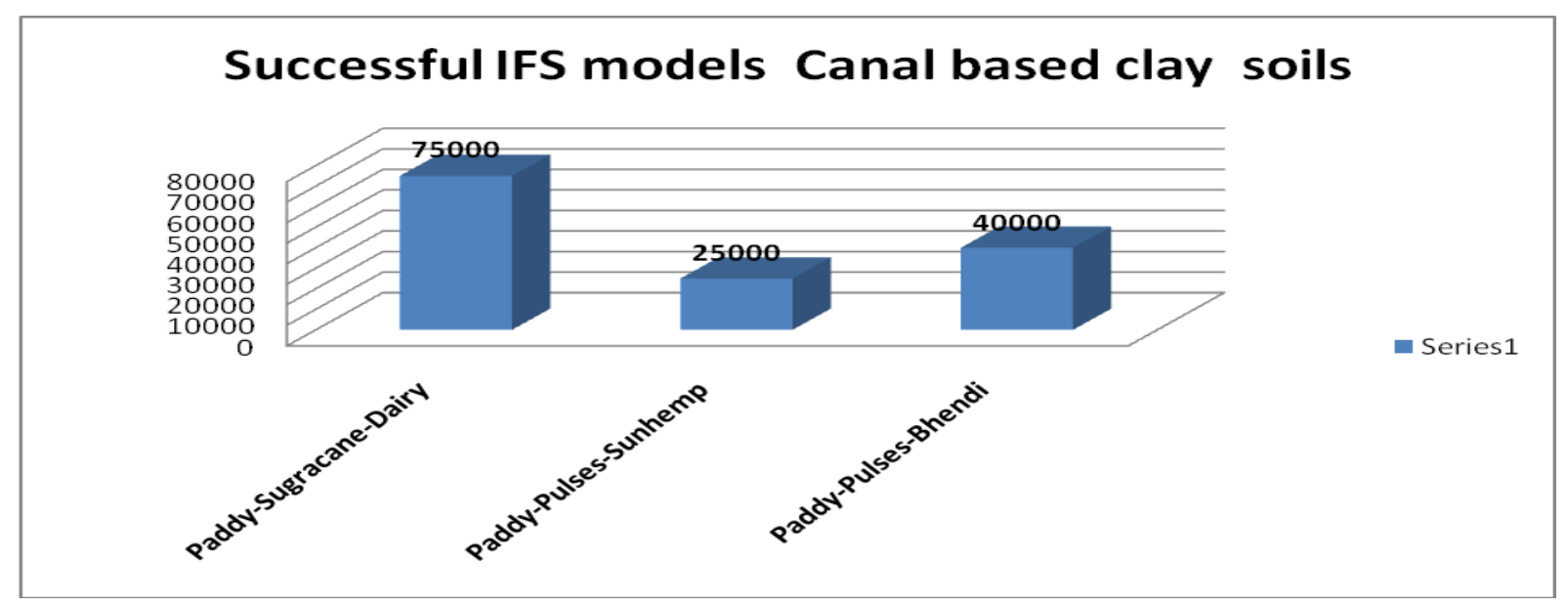


Fig.4

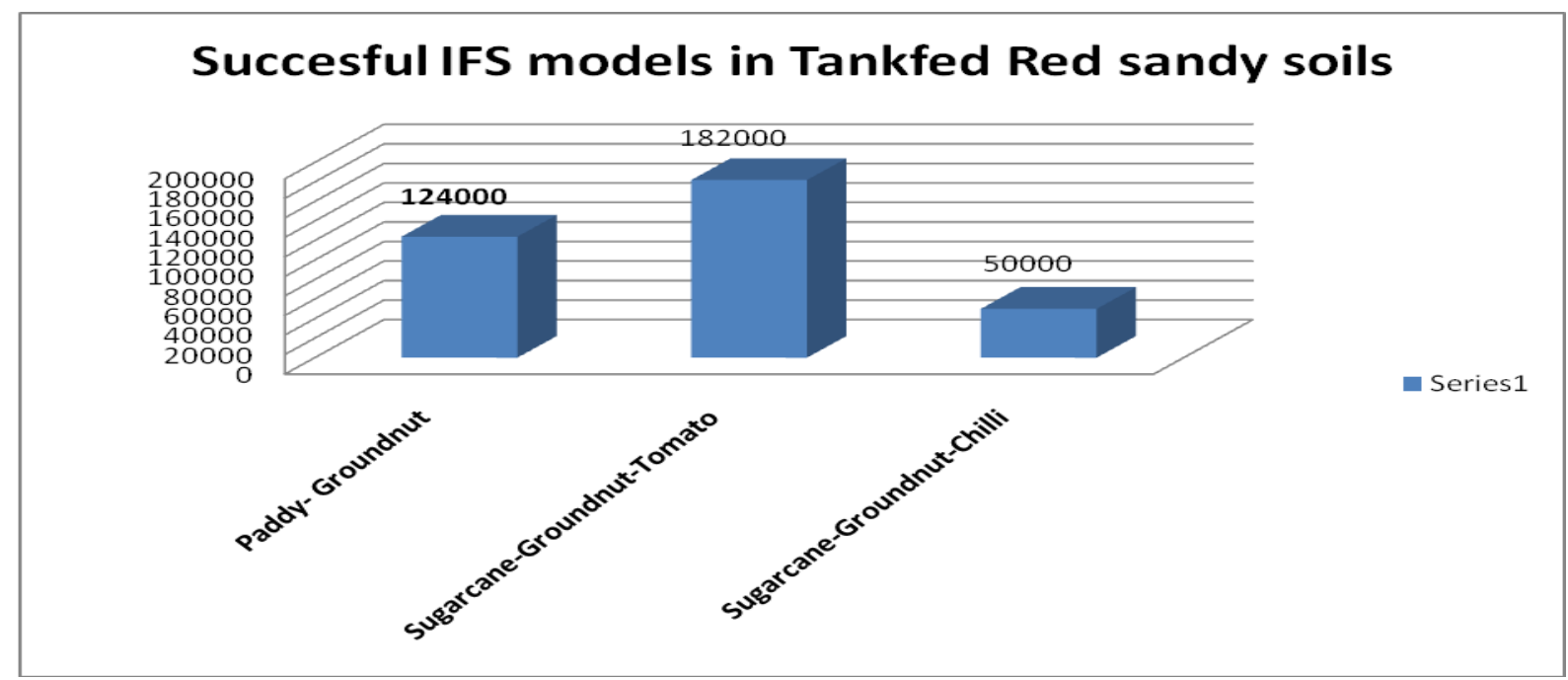

Canal Fed - Sandy loams (Gowripatnam)

Suggestions

Create awareness about IFS models among farmers.

Create awareness about soil testing methods.

Seed should supplied in time.

Minimize the usage of fertilizers and pesticides.

Follow crop rotation in their respective fields.

\section{Dominate IFS models under six major farming situations in the district}

From the below 6 different farming situations 3 top farming systems were identified as per their $\mathrm{B}: \mathrm{C}$ ratio and listed as mentioned in Table 8.

\section{Successful top 3 IFS models under 6 major farming situations in the district}

From all the top 18 farming systems identified as per the $\mathrm{B}: \mathrm{C}$ ratio as mentioned in above table, The top 3 farming situations identified were Sugarcane- Groundnut-Tomato and Paddy Groundnut farming situations from Tankfed Red sandy soils were ranked Ist and
IIIrd respectively and Tomato-Ridge gourd Dairy from Bore well based farming stuation ranked IInd (Table 9).

\section{Inference}

As per the district profile of Visakhapatnam, total farmers of district are 4.56 lakhs as per 2011 censes. Out of the total population of 4.56 lakhs 3.39 lakh (74.34\%) farmers are marginal farmers, 0.69 lakhs (15.13\%) are small farmers, 0.35 lakh (7.6\%) farmers are semi medium farmers, 0.12 Lakh $(2.63 \%)$ are medium farmers and 0.012 lakhs $(0.263 \%)$ farmers are large farmers. Out of the total farming community in the district a negligible $(2.89 \%)$ are Large \& medium farmers and the remaining $97.1 \%$ are marginal, Small, Semi medium farmers.

Majority of the farmers are having little land holding and resources and they are bound to adopt Integrated farming systems spreading in 8 major farming situations covering 3 lakh hectares of area. So the face of the agrarian situation of the district is integrated farming system nature. The farmers are sustaining their livelihoods through these farming systems evolved over years, sustaining 
several vagaries of climate and geographical situations of the district.

Among the all top 18 IFS Models identified across the 6 Major Integrated Farming situations in the district, 1. SugarcaneGroundnut-Tomato 2. Paddy- Groundnut falling under Tank fed Red sand soils farming systems model ranks $1^{\text {st }} \& 3^{\text {rd }}$ respectively and Tomato + Ridge gourd + Dairy falling under Bore well based Red Sandy loams farming systems model ranks $2^{\text {nd }}$.as per B:C ratio (Table 10).

In conclusion, the evolved successful IFS models across the major farming systems over years are to be preserved. Since majority of the farmers in the district are small and marginal, these farming systems will serve as base to provide sustainable in situ livelihoods to the farming communities spreading in remote corners of the district.

\section{References}

Allen, V. G., Baker, M. T., Segarra, E. and Brown, C. P. (2007). Integrated irrigated crop- livestock systems in dry climates. Agronomy Journal 99:346360.

Baishya A, Pathak A K, Bhowmick B C and Ahmed S. 2004. Predominant farming system and alternatives in Assam. Alternate Farming Systems: Enhanced Income and Employment Generation Options for Small and Marginal Farmers, pp 228-37. Singh A K, Gangwar B and Sharma S K (Eds). PDFSR, Modipuram, Uttar Pradesh.

Deka B C, Chatterjee D, Sahoo B, Patra M K, Thirugnanavel A, Kumar R, Krose M, Bhatt B P and Ngachan S V. 2013. Soilwater-plant-animal-society continuum approach for livelihood improvement of Konyaks tribes of Mon in Nagaland, ICAR $\mathrm{RC}$ for $\mathrm{NEH}$
Region, Nagaland Centre, Jharnapani, Medziphema, Nagaland.

Gill M S, Singh J P and Gangwar K S. 2010. Integrated farming system and agriculture sustainability. Indian Journal of Agronomy 54(2): 128-39.

Korikanthimath, V. S. and Manjunath, B. L. (2010). Integrated farming systems for sustainability in agricultural production. Indian Journal of Agronomy 54 (2):140-148.

Kumar R, Deka B C, Thirugnanavel A, Patra M K, Chatterjee D, Borah T R, Barman K K, Rajesha G, Talang H D, Kumar M and Ngachan S V. 2015. Comparative evaluation of different farming system models suitable for small and marginal farmers of Nagaland. Lead paper presented in the National

Kumar S, Singh S S, Meena M K, Shivani and Dey A. 2012. Resource recycling and their management under integrated farming system for lowlands of Bihar. Indian Journal of Agricultural Sciences 82(6): 504-10.

Kumar S, Singh S S, Shivani and Dey A. 2011. Integrated farming systems for Eastern India. Indian Journal of Agronomy 56(4): 297-304.

Rakesh kumar, M K Patra, A Thirugnanavel, Bidyut $\mathrm{C}$ deka, Dibyendu chatterjee, $\mathrm{T}$ R Borah, G Rajesha, H D Talang, S K Ray, Manoj Kumar and Pravin Kumar Upadhyay. 2018. Comparative evaluation of different integrated farming system models for small and marginal farmers under the Eastern Himalayas, Indian Journal of Agricultural Sciences 88 (11): 1722-29, November 2018/Article

Ravisankar N, Pramanik SC, Rai R B, Nawaz S, Biswas T K and Bibi N. 2007. Study on integrated farming system in hilly upland areas of Bay Islands. Indian Journal of Agronomy 52(1): 7-10.

Solaniappan U, Subramanian V and Maruthi 
S G R. 2007. Selection of suitable integrated farming system model for rainfed semi-arid vertic inceptisols in
Tamil Nadu. Indian Journal of Agronomy 52(3): 194-7.

\section{How to cite this article:}

Uma Maheswara Rao, D., N. Sathibabu, K. Tejeswara Rao, N. Rajkumar, V. Gouri and Manasa, G. 2020. Evaluation Study on Viable IFS Models in Visakhapatnam District of Andhra Pradesh. Int.J.Curr.Microbiol.App.Sci. 9(12): 3383-3397. doi: https://doi.org/10.20546/ijcmas.2020.912.402 